\title{
Utilidad de la colangiopancreatografía por resonancia magnética (CPRMN) en pacientes con lesión de vía biliar
}

\author{
Usefulness of magnetic resonance cholangiopancreatography \\ (MRCP) in patients with bile duct injury
}

Diego Fernando Viteri-Cevallos*

\begin{abstract}
Palabras clave: Lesión de vía biliar, colangiopancreatografía, resonancia magnética.
\end{abstract}

Key words: Bile duct injury, cholangiopancreatography, magnetic resonance.

\section{* Servicio de}

Gastrocirugía del Hospital de Especialidades del

Centro Médico Nacional Siglo XXI. Instituto

Mexicano del Seguro Social.

Recibido: 18/04/2016 Aceptado: 12/07/2017

\section{RESUMEN}

Introducción: Las estenosis biliares benignas incluyen diversas entidades clínicas; el grupo más importante corresponde a quienes presentan una lesión de vía biliar postoperatoria (LVB), cuya incidencia se ha elevado desde el $0.1-0.2 \%$ al $0.4-0.7 \%$ a partir de la colecistectomía laparoscópica, aunque es similar a la de la cirugía abierta en manos de cirujanos expertos. Dentro del diagnóstico, la CPRMN ha demostrado ser comparable con la CPRE (colangiopancreatografía retrógrada endoscópica) en la localización y extensión de las LVB extrahepáticas, con una sensibilidad del 91 al 100\%. Material y métodos: Se realizó un estudio de correlación retrospectivo en el Servicio de Gastrocirugía del HE, CMN Siglo XXI. Se observó la concordancia (kappa de Cohen) entre la CPRMN con los hallazgos quirúrgicos en pacientes intervenidos por LVB, así como su validación como herramienta diagnóstica. Resultados: Se incluyeron 40 pacientes en este estudio. Se encontró que la concordancia global para la presencia de LVB fue 0.55 ; la hallada en relación con el nivel anatómico de la LVB fue de 0.59; para determinar si la LVB era completa o parcial, el kappa fue de 0.39 , y para la presencia de colecciones intrabdominales, de 0.59 (0.32-0.86). En relación con la validez diagnóstica, se observó que para fijar en forma global la presencia de una LVB, la sensibilidad (S) fue de $88.9 \%$ y la especificidad (E) de $80 \%$; para establecer el nivel de lesión preoperatorio, la S fue de $84 \%$ y la E de $26 \%$. Conclusiones: Se concluye que existe una correlación diagnóstica moderada entre la CPRMN y los hallazgos quirúrgicos en pacientes intervenidos por lesión de vía biliar.

\section{ABSTRACT}

Introduction: Benign biliary strictures include several clinical entities; the most important group is for those who had a postoperative bile duct injury (BDI), the incidence of which has risen from $0.1-0.2 \%$ to $0.4-0.7 \%$ from laparoscopic cholecystectomy, although the incidence is similar to that of open surgery in the hands of expert surgeons. Within the diagnostic, MRCP has proven to be comparable with ERCP in the location and extent of extrahepatic LVB, with a sensitivity of 91 to $100 \%$. Material and methods: A retrospective correlation was performed in the Gastrosurgery Service of HE Siglo XXI. The correlation (Cohen's kappa) between the MRCP with surgical findings in patients undergoing LVB was observed, as well as its validation as a diagnostic tool. Results: Forty patients were included in this study. It was found that the overall correlation for the presence of BDI was 0.55; the one identified in relation to the anatomical level of the LVB was 0.59; to determine whether the BDI was complete or partial, kappa was of 0.39 , and for the presence of intraabdominal collections, 0.59 (0.32 to 0.86). Regarding the diagnostic accuracy, it was found that to determine globally the presence of a BDI, the sensitivity (S) was $88.9 \%$ and the specificity (Sp) of $80 \%$; to determine the level of preoperative lesion, $S$ was $84 \%$ and Sp 26\%. Conclusions: We conclude that there is a moderate correlation between MRCP diagnostic and surgical findings in patients undergoing surgery for bile duct injury.

\section{INTRODUCCIÓN}

L as estenosis biliares benignas (EBB) incluyen varias patologías que se pueden caracterizar por abdomen agudo postoperatorio, datos de obstrucción o fuga biliar con o sin evidencia de peritonitis, que ocurren secundarios a una lesión intraoperatoria, una anastomosis por la reparación quirúrgica del conducto biliar, trasplante hepático o estenosis inflamatorias postoperatorias. El tratamiento eficaz es necesario con el fin de evitar el deterioro de la función hepática, colangitis, formación de abscesos y sepsis. ${ }^{1,2}$ 
Las causas más frecuentes de estenosis benignas del árbol biliar pueden estar relacionadas con lesiones de vía biliar postoperatorias (LVB), secundarias a una colecistectomía, resección gástrica o hepática, derivación porto-cava o anastomosis bilio-entérica. ${ }^{3}$

Los factores de riesgo asociados con LVB son obesidad, edad avanzada, género masculino, inflamación severa, anatomía aberrante y hemorragia. Además, las lesiones térmicas en la pared ductal por la propagación del calor pueden producir una estenosis progresiva de la vía biliar. ${ }^{4,5}$

Desde la introducción de la colecistectomía laparoscópica en la década de 1990, la incidencia de las lesiones biliares se ha duplicado de 0.2 a $0.4 \%$. Otros reportes han demostrado una elevación de 0.1 a $0.7 \% .^{6-9}$ El patrón de las LVB ha cambiado, con lesiones más proximales y la isquemia relacionada con lesiones vasculares o por energía. ${ }^{10}$

La presentación clínica inicial es secundaria a la obstrucción aguda del conducto biliar y se vuelve clínicamente evidente con ictericia, alteraciones a nivel de la función hepática, fiebre o datos de sepsis. En pacientes con fuga biliar $y / 0$ fístulas, el cuadro se presenta generalmente con dolor abdominal y elevación de marcadores inflamatorios en el postoperatorio inmediato. ${ }^{11}$

El reconocimiento intraoperatorio de una LVB permite que un cirujano hepatobiliar evalúe la gravedad, teniendo en cuenta que hasta el $90 \%$ de las lesiones no son diagnosticadas durante la cirugía. ${ }^{12}$

Dentro del diagnóstico, la colangiopancreatografía por resonancia magnética (CPRMN) es una alternativa no invasiva y fiable para la evaluación de la vía biliar. Ha demostrado ser comparable con la CPRE en la localización y extensión de estenosis a nivel extrahepático, con una sensibilidad (S) entre 91 y 100\%. Otros reportes mencionan que la exactitud de la CPRMN frente a la CPRE para valorar el tipo y lugar de la obstrucción es de 76 versus $72 \% .{ }^{13-16}$ En otro estudio, Kaltenthaler y sus colaboradores compararon la CPRMN con la CPRE en pacientes con dilatación de la vía biliar, y obtuvieron una $S$ de 87 a 100\% y una especificidad (E) de 91 a 100\%; para valorar estenosis encontraron una S de $100 \%$ y E de 98 a $99 \% .{ }^{17}$

En un estudio realizado por Busel y su grupo, en el que se comparó al ultrasonido
(US) con la CPRMN en pacientes con ictericia obstructiva, se encontró que el US mostró una $\mathrm{S}$ de $58.33 \%$ y $\mathrm{E}$ de $82.5 \%$; en cambio, la CPRMN presentó una $\mathrm{S}$ de $95.83 \%$ y $\mathrm{E}$ de $87.5 \%$, con una capacidad alta para determinar a los pacientes sanos con una prueba negativa (VPN de $97.22 \%){ }^{18}$

Con el fin de definir el tipo de LVB, se han propuesto varias clasificaciones, pero ninguna es universalmente aceptada. La clasificación de Bismuth se originó en la época de la cirugía abierta; se basa en el nivel más distal en que la mucosa biliar se encuentra sana proximal al sitio de la lesión o estenosis; tiene por objetivo ayudar al cirujano a elegir la técnica apropiada para la reparación y tiene una buena correlación con el resultado final después de la reparación quirúrgica. ${ }^{9,19}$

Strasberg propuso una clasificación mucho más amplia mediante la inclusión de otros tipos de lesiones extrahepáticas laparoscópicas, que incluyen fugas de conductos accesorios o del cístico; ésta es la más útil y recomendada en la actualidad. ${ }^{20}$ La Asociación Europea de Cirugía Endoscópica (EAES) elaboró una nueva clasificación con el objetivo de tener en cuenta el carácter cambiante de las lesiones sufridas desde la introducción de la colecistectomía laparoscópica y combinar todos los elementos existentes en la mayoría de las clasificaciones utilizadas hasta la fecha; esto puede conducir a una determinación más precisa de la verdadera incidencia de la LVB. Se valora el nivel anatómico similar a clasificaciones previas, lesión vascular asociada, extensión de la lesión, mecanismo y tiempo de detección. ${ }^{10}$

El tratamiento depende del tiempo de reconocimiento de la lesión, la extensión, la condición del paciente y la disponibilidad de cirujanos hepatobiliares experimentados.

La detección inmediata y reparación de la lesión se asocian con un mejor resultado, siempre y cuando el cirujano que la efectúe tenga una experiencia amplia en este tipo de complicaciones graves. Existen estudios en los que no encontraron una gran diferencia entre la reparación inmediata en comparación con el manejo conservador inicial como, por ejemplo, el aspecto nutricional, hidroelectrolítico, etcétera, y una cirugía reconstructiva posterior. ${ }^{9}$

La mayoría de los pacientes requieren cirugía reconstructiva; en casos seleccionados 
con lesiones menores, la terapia endoscópica es exitosa. Las estrategias terapéuticas han cambiado en pacientes que inicialmente se tratan con endoscopia $y$, posteriormente, de manera quirúrgica. ${ }^{21}$ Del 22 al $36 \%$ de quienes se someten a una cirugía adicional tendrán estenosis después de cada procedimiento. ${ }^{22}$ Los pacientes presentan un alto riesgo de desarrollar complicaciones como fuga anastomótica, hemorragia, colangitis, litiasis y estenosis, las cuales pueden ir del 3 al $43 \% .^{23,24}$

Durante muchos años, la CPRE ha sido la primera opción para el alivio de la obstrucción biliar en la mayoría de las ocasiones. El uso de stents biliares es un tratamiento común y generalizado tanto para el drenaje biliar inicial como para dilataciones progresivas en forma tardía. ${ }^{25,26}$

Los objetivos del presente trabajo fueron conocer la correlación diagnóstica entre la CPRMN y los hallazgos quirúrgicos en pacientes intervenidos por LVB, establecer la eficacia de la CPRMN como herramienta diagnóstica y saber la frecuencia, tipo y manejo quirúrgico realizado.

La hipótesis de nuestra investigación fue que existe una muy buena correlación diagnóstica (índice kappa 0.81-1) entre la CPRMN y los hallazgos quirúrgicos en pacientes intervenidos por diagnóstico de LVB.

\section{MATERIAL Y MÉTODOS}

Se realizó un estudio de correlación retrospectivo en el Servicio de Gastrocirugía del Hospital de Especialidades del Centro Médico Nacional Siglo XXI, entre agosto de 2012 y julio de 2013, en los pacientes ingresados al Servicio de Gastrocirugía con LVB, con CPRMN preoperatoria y que fueron intervenidos quirúrgicamente.

Para el desarrollo de esta investigación fueron incluidos todos los pacientes con el diagnóstico de sospecha de lesión de vía biliar. Posteriormente, se seleccionaron los expedientes clínicos y radiológicos de aquéllos en quienes se realizó la CPRMN y fueron intervenidos quirúrgicamente; de ellos, con base en la hoja de recolección de datos, se obtuvo la información correspondiente a cada historia.

Los datos registrados fueron sistematizados para aplicar las pruebas estadísticas que permitieron cumplir los objetivos planteados en este estudio.
El análisis de variables se realizó con base en la variable dependiente (correlación radiológica-quirúrgica entre la CPRMN y los hallazgos quirúrgicos en LVB) y las variables independientes: clasificación de LVB preoperatoria $(\mathrm{CPRMN})$ y postoperatoria (hallazgos quirúrgicos), categorizadas con base en la clasificación de Strasberg, extensión de LVB completa o parcial, presencia de colecciones intraabdominales y la dilatación biliar evaluadas antes y después de la cirugía.

Los criterios de inclusión fueron los pacientes que ingresaron al Servicio de Gastrocirugía del Hospital de Especialidades del Centro Médico Nacional Siglo XXI con diagnóstico de LVB, en los cuales, dentro de su protocolo de estudio, se realizó CPRMN y fueron intervenidos quirúrgicamente. Se excluyeron quienes tuvieron un diagnóstico de origen maligno a nivel de vía biliar o no cumplieron los criterios previos.

Los descriptivos de las variables categóricas (clasificación preoperatoria y postoperatoria, extensión de la lesión) están expresados mediante frecuencias absolutas y porcentajes, y los de las variables cuantitativas (edad), a través de medidas de tendencia central (media) y dispersión (desviación estándar y rango).

Para el cálculo de la correlación entre el diagnóstico por CPRMN y los hallazgos transoperatorios, se utilizó el índice kappa de Cohen. Este índice de concordancia entre observadores (radiológico versus quirúrgico) representa una aportación que incorpora en su fórmula una corrección que excluye la concordancia debida exclusivamente al azar, misma que está relacionada con las distribuciones marginales (Cuadro I).27,28

Además, para el análisis estadístico de la CPRMN como prueba diagnóstica en pacientes con LVB, se obtuvieron los siguientes índices de probabilidad (S, E, VPP, VPN, RV+/-) mediante tablas de $2 \times 2 .{ }^{29}$ Se utilizó como patrón de oro para la investigación el hallazgo quirúrgico con base en el nivel y tipo de LVB. Fue identificado el tipo de tratamiento quirúrgico realizado en relación con el diagnóstico postoperatorio establecido.

Para la sistematización, procesamiento y análisis de datos, se utilizaron los programas Microsoft Excel (Microsoft Office para Win- 
dows versión 2011) y SPSS (Windows español versión 11.5).

\section{RESULTADOS}

Durante el periodo en que se realizó la investigación, 65 pacientes acudieron al Servicio de Urgencias del Hospital de Especialidades del Centro Médico Nacional Siglo XXI por sospecha diagnóstica de LVB; de ellos, 40 cumplieron los criterios de inclusión e ingresaron al estudio. Se

\begin{tabular}{cc}
\multicolumn{2}{c}{$\begin{array}{c}\text { Cuadro I. Interpretación del índice } \\
\text { kappa de Cohen. }\end{array}$} \\
\hline Valor kappa & Fuerza de concordancia \\
\hline$<20$ & Pobre \\
$0.21-0.40$ & Débil \\
$0.41-0.60$ & Moderada \\
$0.61-0.80$ & Buena \\
$0.81-1.00$ & Muy buena \\
\hline
\end{tabular}

Cuadro II. Datos demográficos de los pacientes con LVB.

\begin{tabular}{lrl} 
Datos & Núm. (\%) \\
\hline Edad, media (DE), mediana (rango) & $47.8(17.7), 45.5(19-92)$ \\
Abordaje & 11 & $(27.5)$ \\
Abierto & 29 & $(72.5)$ \\
Laparoscópico & 9 & $(22.5)$ \\
Urgente & 31 & $(77.5)$ \\
Programado & 11 & $(27.5)$ \\
Conversión a cirugía abierta & & \\
Tiempo de detección & 5 & $(12.5)$ \\
Durante la colecistectomía & 35 & $(87.5)$ \\
Postoperatorio & & \\
Estudios preoperatorios & 16 & $(40)$ \\
CPRE & 7 & $(17.5)$ \\
TC & 17 & $(42.5)$ \\
US & 3 & $(7.5)$ \\
Colangiografía & & \\
ASA & 2 & $(5)$ \\
1 & 26 & $(65)$ \\
2 & 10 & $(25)$ \\
3 & 2 & $(5)$
\end{tabular}

incluyeron 11 hombres (27.5\%) y 29 mujeres (72.5\%), con una edad media de 47 años ( \pm 17 años).

En cinco pacientes $(12.5 \%)$ se reconoció la LVB durante la colecistectomía; de ellos, a tres se les colocó sonda en T, a uno, sondas transhepáticas, y a otro se le realizó laparotomía y derivación de la vía biliar.

Al momento del ingreso a nuestro servicio, el estado clínico inicial fue fuga biliar en $\mathbf{1 6}$ pacientes $(40 \%)$, reconocida por gasto a través de una herida o drenaje mayor a $100 \mathrm{~cm}^{3} / 24$ horas; ictericia en 13 (32.5\%), con bilirrubina total mayor a $2.6 \mathrm{mg} / \mathrm{dl}$; sepsis intraabdominal en ocho (20\%), y tres asintomáticos (7.5\%) (Cuadro II).

En nuestra cohorte, las LVB se clasificaron según Strasberg; de ellas, las tipo E2 (B II) y E3 (B III) se presentaron en el $65 \%$ de los pacientes; además, hubo tres (7.5\%) en quienes se presentó una fuga biliar relacionada con una lesión Strasberg A (Cuadro III).

En nuestra institución, por ser un centro de referencia, los pacientes fueron enviados de otros hospitales por sospecha de LVB; al ser admitidos, fueron estabilizados y protocolizados; al confirmarse la LVB se iniciaron antibióticos, de ser necesario, y se decidió su tratamiento quirúrgico.

Los procedimientos realizados fueron: hepático-yeyuno anastomosis en el $37.5 \%$, técnica de Hepp-Couinaud en el $32.5 \%$, exploración de vía biliar y colocación de sonda en T en el $7.5 \%$, laparotomía con drenaje en el $15 \%$, colédoco-duodeno anastomosis en el $5 \%$ y doble anastomosis en $2.5 \%$. Se presentaron complicaciones en el $54 \%$ de los pacientes; las más comunes fueron la fuga biliar (48.6\%), que remitió con manejo conservador en el 95\% de los casos, infección de herida quirúrgica en el $10.8 \%$ y absceso intraabdominal en el $8.1 \%$ (Cuadro IV).

Se obtuvo el valor kappa (concordancia entre observadores), con los siguientes resultados: la concordancia global para la presencia de LVB fue 0.55 , la encontrada en relación al nivel anatómico de la LVB (clasificación de Bismuth) fue de 0.59 (0.37-0.80); para determinar si la LVB era completa o parcial, el kappa fue de 0.39 (0.16-0.62); la presencia de colecciones intraabdominales tuvo un valor kappa de 0.59 
Cuadro III. Clasificación de Strasberg en pacientes con LVB.

\begin{tabular}{llc} 
Clasificación de la lesión & Descripción & Núm. (\%) \\
\hline Sin lesión & & $1(2.5)$ \\
A & Fuga biliar del conducto cístico o de un conducto menor & $3(7.5)$ \\
B & Lesión de un conducto hepático derecho aberrante & 0 \\
C & Fuga biliar de un conducto sin comunicación & 0 \\
D & Lesión lateral de un conducto biliar extrahepático & 0 \\
E1 & Estenosis del conducto hepático común a más de dos centímetros de la confluencia biliar & $5(12.5)$ \\
E2 & Con un trayecto corto menor a dos centímetros & $14(35)$ \\
E3 & Sólo el techo de la confluencia biliar está intacto & $12(30)$ \\
E4 & Se interrumpe la confluencia biliar & $4(10)$ \\
E5 & Estenosis del conducto hepático asociado a una estenosis en una rama accesoria derecha & $1(2.5)$
\end{tabular}

Cuadro IV. Complicaciones postoperatorias en pacientes postoperados de derivación biliodigestiva.

\begin{tabular}{lrl} 
Complicación & Núm. (\%) \\
\hline Fuga biliar & $18(48.6)$ \\
Infección de la herida quirúrgica & $4 \quad(10.8)$ \\
Absceso intraabdominal & $3(8.1)$ \\
Coledocolitiasis residual & $2(5.4)$ \\
Hernia incisional & $1(2.7)$ \\
Neumonía & $1(2.7)$ \\
Sangrado postoperatorio & $1(2.7)$
\end{tabular}

Cuadro V. Correlación de la CPRMN vs. hallazgos quirúrgicos en relación con el nivel de LVB.

\begin{tabular}{|c|c|c|c|c|c|c|c|c|}
\hline & & \multicolumn{6}{|c|}{ Tratamiento quirúrgico } & \multirow[b]{2}{*}{ Tota } \\
\hline & & E1 & E2 & E3 & E4 & E5 & SL & \\
\hline \multirow{6}{*}{$\mathrm{RX}$} & E1 & 3 & 3 & 0 & 0 & 0 & 0 & 6 \\
\hline & E2 & 0 & 10 & 4 & 1 & 0 & 0 & 15 \\
\hline & E3 & 0 & 1 & 6 & 0 & 0 & 0 & 7 \\
\hline & E4 & 0 & 0 & 0 & 2 & 0 & 0 & 2 \\
\hline & E5 & 0 & 0 & 0 & 0 & 1 & 0 & 1 \\
\hline & SL & 2 & 0 & 2 & 1 & 0 & 4 & 9 \\
\hline Total & & 5 & 14 & 12 & 4 & 1 & 4 & 40 \\
\hline
\end{tabular}

(0.32-0.86), y en relación con la presencia de dilatación extrahepática, kappa de 0.1 (0.14-0.38) e intrahepática de 0.4 (0.11-0.68) (Cuadro V).
Mediante tablas de $2 \times 2$ se evaluó a la CPRMN como prueba diagnóstica para LVB; los resultados se muestran en el cuadro VI.

\section{DISCUSIÓN}

La estenosis postoperatoria de vía biliar, cuya causa principal es la lesión durante la colecistectomía abierta o laparoscópica (LVB), es un problema de salud importante, con una incidencia reportada del $0.4-0.7 \% .^{9}$ Tiene un impacto desfavorable en la supervivencia de los pacientes, un alto costo de su manejo y complicaciones a largo plazo.

El mecanismo de la lesión, los intentos previos de reparación, el riesgo quirúrgico y el estado de salud influyen en la decisión terapéutica. Es necesario un abordaje multidisciplinario para lograr un manejo integral en cada paciente. ${ }^{30}$ Dentro del protocolo diagnóstico se encuentra el uso de la CPRMN, herramienta radiológica que nos permite determinar la magnitud de la LVB y su posible tratamiento quirúrgico.

En el presente trabajo ingresaron 40 pacientes a la investigación, con una edad promedio de 47 años y mayor prevalencia en el género femenino $(72 \%)$, resultado similar a estudios realizados previamente, donde encontraron una media de edad de 47 años y $65 \%$ de mujeres. ${ }^{31}$

Es importante mencionar que en el 5\% de los pacientes, la detección de la lesión fue intraoperatoria, a diferencia de estudios que señalan que del 10 al $25 \%$ de las LVB se reconocen en el momento de la cirugía; esta diferencia 
Cuadro VI. Validez diagnóstica de la CPRMN en pacientes con LVB.

\begin{tabular}{lrlrl} 
Índice & Diagnóstico global LVB & \multicolumn{1}{c}{ Nivel de LVB } \\
\hline Sensibilidad & 88.9 & $(73.92-96.82)$ & $84(63.9-95.3)$ \\
Especificidad & 80 & $(28.81-96.7)$ & 26.67 & $(7.9-55.09)$ \\
LR + & 4.44 & $(0.77-25.75)$ & $1.15(0.81-1.63)$ \\
LR - & 0.14 & $(0.05-0.39)$ & $0.60(0.18-2.05)$ \\
VPP & 96.9 & $(84.1-99.4)$ & $65.6(46.8-81.4)$ \\
VPN & $50(16.1-83.9)$ & $50(16-83.9)$
\end{tabular}

puede estar dada porque nuestro hospital es un centro de referencia al que llegan los pacientes complicados tras una derivación biliodigestiva o en los que la detección fue postoperatoria. ${ }^{12,31}$

Un estudio realizado por Sahajpal y sus colaboradores menciona que las manifestaciones clínicas más importantes por las que acuden los pacientes son ictericia o datos de fuga biliar; en esta investigación se encontró que el 75\% de los pacientes presentaron estos datos clínicos. ${ }^{31}$

Este trabajo demostró que la CPRMN tiene una fuerza de concordancia moderada en relación con los hallazgos en pacientes intervenidos quirúrgicamente, tanto para determinar la presencia de una LVB como para establecer el nivel anatómico de lesión, lo que sugiere que es un estudio útil para evaluar preoperatoriamente un paciente con sospecha de LVB en el que se decide tratamiento quirúrgico, aunque la correlación podría ser mejor si existiera un mayor entrenamiento a nivel radiológico para interpretar adecuadamente los hallazgos por imagen y se podría definir en forma más clara el tratamiento quirúrgico en pacientes con sospecha de LVB.

En investigaciones previas realizadas en lo que se refiere a la utilidad como prueba diagnóstica de la CPRMN en LVB, existen estudios que demuestran una $\mathrm{S}$ de 91 a $100 \%{ }^{14}$ en otro estudio se encontró una $\mathrm{S}$ de $93.8 \%$, $\mathrm{E}$ de $93.8 \%$ y eficacia de $89 \%{ }^{32}$ En este trabajo, para determinar en forma global la presencia de una LVB, la sensibilidad y especificidad fueron altas, con una aceptable probabilidad de que un sujeto con resultado positivo tuviera la enfermedad y con cuatro veces más probabilidad de encontrar un paciente con LVB al realizar este estudio $(L R+)$.
Al momento de evaluar la CPRMN para determinar el nivel anatómico de LVB, se encontró una sensibilidad alta con una especificidad baja, lo que demuestra que es una prueba recomendable para detectar la presencia de una lesión; sin embargo, según los resultados obtenidos, es poco útil para conocer el nivel de lesión, lo cual es necesario para saber el tipo de procedimiento quirúrgico que se va a realizar. Estos resultados difieren de estudios previos por la poca especificidad para determinar el nivel de LVB.

Es importante indicar que este trabajo presenta limitaciones, ya que hubo un porcentaje de datos que no se pudo obtener por la falta de información o por la ausencia de expedientes en algunos casos.

\section{CONCLUSIONES}

En este estudio, por los resultados obtenidos, se concluye que existe una correlación diagnóstica moderada entre la CPRMN y los hallazgos quirúrgicos en pacientes intervenidos por LVB, con una validez diagnóstica aceptable, aunque con menor capacidad para evaluar preoperatoriamente el lugar anatómico de la lesión.

\section{REFERENCIAS}

1. Parikh P, Lillemoe K. Choledochal cyst and bening biliary strictures. In: Zinner M, ed. Maingot's abdominal operations. 12th ed. Chicago: McGrawHill; 2013. pp. 1029-1059.

2. Krokidis M, Orgera G, Rossi M, Matteoli M, Hatzidakis A. Interventional radiology in the management of benign biliary stenoses, biliary leaks and fistulas: a pictorial review. Insights Imaging. 2013; 4: 77-84

3. Judah JR, Draganov PV. Endoscopic therapy of benign biliary strictures. World J Gastroenterol. 2007; 13: 3531-3539.

4. Wu YV, Linehan DC. Bile duct injuries in the era of laparoscopic cholecystectomies. Surg Clin North Am. 2010; 90: 787-802.

5. Lillemoe KD. Benign post-operative bile duct strictures. Baillieres Clin Gastroenterol. 1997; 11: 749-779.

6. Flum DR, Cheadle A, Prela C, Dellinger EP, Chan L. Bile duct injury during cholecystectomy and survival in medicare beneficiaries. JAMA. 2003; 290: 2168-2173.

7. Nuzzo G, Giuliante F, Giovannini I, Ardito F, D'Acapito $\mathrm{F}$, Vellone $\mathrm{M}$, et al. Bile duct injury during laparoscopic cholecystectomy: results of an Italian national survey on 56 591 cholecystectomies. Arch Surg. 2005; 140: 986-992.

8. Waage A, Nilsson M. latrogenic bile duct injury: a population-based study of 152776 cholecystectomies in the Swedish Inpatient Registry. Arch Surg. 2006; 141: 1207-1213. 
9. Lau WY, Lai EC. Classification of iatrogenic bile duct injury. Hepatobiliary Pancreat Dis Int. 2007; 6: 459-463.

10. Fingerhut A, Dziri C, Garden OJ, Gouma D, Millat B, Neugebauer E, et al. ATOM, the all-inclusive, nominal EAES classification of bile duct injuries during cholecystectomy. Surg Endosc. 2013; 27: 4608-4619.

11. Doctor N, Dooley JS, Dick R, Watkinson A, Rolles K, Davidson BR. Multidisciplinary approach to biliary complications of laparoscopic cholecystectomy. Br J Surg. 1998; 85: 627-632.

12. Connor S, Garden OJ. Bile duct injury in the era of laparoscopic cholecystectomy. Br J Surg. 2006; 93: 158-168.

13. Girometti R, Brondani G, Cereser L, Como G, Del Pin $M$, Bazzocchi $M$, et al. Post-cholecystectomy syndrome: spectrum of biliary findings at magnetic resonance cholangiopancreatography. Br J Radiol. 2010; 83: 351-361.

14. Halefoglu AM. Magnetic resonance cholangiopancreatography: a useful tool in the evaluation of pancreatic and biliary disorders. World J Gastroenterol. 2007; 13: 2529-2534.

15. Rossi M, Salvatori F, Giglio L, Fanelli F, Cantisani V, Rossi $\mathrm{P}$, et al. Interventional radiology techniques in the treatment of complications due to videolaparoscopic cholecystectomy. Radiol Med. 2003; 103: 384-395.

16. Gibson RN, Vincent JM, Speer T, Collier NA, Noack $\mathrm{K}$. Accuracy of computed tomographic intravenous cholangiography (CT-IVC) with iotroxate in the detection of choledocholithiasis. Eur Radiol. 2005; 15: 1634-1642.

17. Busel MD, Pérez ML, Arroyo PA, Ortega TD, Niedmann EJ, Palavecino RP, et al. Colangiorresonancia (CPRM) vs ultrasonido (US) focalizado en pacientes con ictericia o sospecha de obstrucción de la vía biliar: resultados preliminares. Rev Chil Radiol. 2003; 9: 173-181.

18. Kaltenthaler EC, Walters SJ, Chilcott J, Blakeborough A, Vergel YB, Thomas S. MRCP compared to diagnostic ERCP for diagnosis when biliary obstruction is suspected: a systematic review. BMC Med Imaging. 2006; 6: 9.

19. Bismuth H, Majno PE. Biliary strictures: classification based on the principles of surgical treatment. World J Surg. 2001; 25: 1241-1244.

20. Strasberg SM, Hertl M, Soper NJ. An analysis of the problem of biliary injury during laparoscopic cholecystectomy. J Am Coll Surg. 1995; 180: 101-125.
21. Jayasundara JA, de Silva WM, Pathirana AA. Changing clinical profile, management strategies and outcome of patients with biliary tract injuries at a tertiary care center in Sri Lanka. Hepatobiliary Pancreat Dis Int. 2011; 10: 526-532.

22. Gwon DI, Ko GY, Ko HK, Yoon HK, Sung KB. Percutaneous transhepatic treatment using retrievable covered stents in patients with benign biliary strictures: mid-term outcomes in 68 patients. Dig Dis Sci. 2013; 58: 3270-3279.

23. Nealon WH, Urrutia F. Long-term follow-up after bilioenteric anastomosis for benign bile duct stricture. Ann Surg. 1996; 223: 639-645; discussion 645-648.

24. Boraschi P, Nonati F. Biliary-enteric anastomoses: spectrum of findings on Gd-EOB-DTPA-enhanced MR cholangiography. Abdom Imaging 2013;38:1351-1359.

25. García-Cano J. Endoscopic management of benign biliary strictures. Curr Gastroenterol Rep. 2013; 15: 336.

26. Nagano Y, Togo S, Tanaka K, Masui H, Endo I, Sekido H, et al. Risk factors and management of bile leakage after hepatic resection. World J Surg. 2003; 27: 695-698.

27. Cerda LJ, Villarroel PL. Evaluación de la concordancia inter-observador en investigación pediátrica: Coeficiente de Kappa. Rev Chil Pediatr. 2008; 79: 54-58.

28. Warrens MJ. On the equivalence of Cohen's kappa and the Hubert-Arabie adjusted rand index. Journal of Classification. 2008; 25: 177-183.

29. Knottnerus JA, van Weel C, Muris JW. Evaluation of diagnostic procedures. BMJ. 2002; 324: 477-480.

30. Mercado MA, Domínguez I. Classification and management of bile duct injuries. World J Gastrointest Surg. 2011; 3: 43-48.

31. Sahajpal AK, Chow SC, Dixon E, Greig PD, Gallinger S, Wei AC. Bile duct injuries associated with laparoscopic cholecystectomy: timing of repair and long-term outcomes. Arch Surg. 2010; 145: 757-763.

32. Zhong L, Yao QY, Li L, Xu JR. Imaging diagnosis of pancreato-biliary diseases: a control study. World J Gastroenterol. 2003; 9: 2824-2827.

Correspondencia:

Dr. Diego Fernando Viteri Cevallos

Hospital de la Policía de Quito Núm. 1, Mariana de Jesús y Av. Occidental, Quito-Ecuador.

Tel. 5932345853

Cel. 593958989068

E-mail: diegoviteri83@hotmail.com 